Elizabeth Calatrava

Isabel Casanovas

Carla Foronda

Fernando Cobo

\title{
Joint infection due to Elizabethkingia miricola
}

Department of Microbiology and Instituto Biosanitario de Granada, Hospital Virgen de las Nieves. Granada, Spain

\section{Article history}

Received: 14 October 2019; Revision Requested: 5 November 2019; Revision Received: 10 December 2019; Accepted: 7 January 2020; Published: 10 March 2020

\section{Sir,}

Elizabethkingia miricola is a non-fermenting Gram-negative rod, non-motile and non-spore-forming which was firstly described in 2003 when it was isolated from condensation water on the space station MIR [1]. Initially named Chryseobacterium miricola, it was reclassified along with Chryseobacterium meningosepticum into the new genus Elizabethkingia, this was due to the phylogenetic analysis, based on the sequencing of the 16S rRNA gene [2]. E. miricola has been demonstrated to be pathogenic, with reports of bacteremia, sepsis and pulmonary abscesses [3-5]. To our best knowledge, we report the first case of infection in a native joint due to E. miricola.

A 52-years-old immunocompetent woman came to the Emergency Department of our hospital due to non-favorable evolution of a catastrophic right foot caused by a traffic accident. The diagnosis was made by means of a foot $\mathrm{x}$-ray and it was observed that the affected bones were the calcaneus, talus, scaphoid and cuboid. Patient refers poor evolution of skin injuries during a month with pain that has slightly subsided. The clinical history was unremarkable and no underlying diseases were reported. No antibiotic treatment during that month was administered, and only wound cures every two days were performed. Due to the infectious aspect of the right ankle, a surgical procedure with extensive debridement was then carried out; also, five bone biopsies from different sites were taken and sent to the microbiology laboratory for culture. The patient did not have indwelling devices or invasive catheters before infection developed. After processing, the samples were inoculated in blood agar (both aerobic and anaerobic) (BD Columbia Agar $5 \%$ Sheepblood ${ }^{\circledR}$, Becton Dickinson), chocolate agar (BD Choco agar, Becton Dickinson) and thioglycollate broth (BD Fluid Thio-

Correspondence:

Dr. Fernando Cobo, MD, PhD

Department of Microbiology, Hospital Virgen de las Nieves

Avda Fuerzas Armadas, 218014 Granada, Spain

Phone: +34958020364 - Fax: +34958241245

E-mail: fernando.cobo.sspa@juntadeandalucia.es glycollate Medium, Becton Dickinson). All media were incubated at $37^{\circ} \mathrm{C}$ during 5 days.

Direct Gram staining of the samples showed abundant Gram-negative bacilli, and on the first day of incubation growth of grey colonies in pure culture was observed on all plates above mentioned. The oxidase test was positive (0xidase Reagent Droppers, Becton Dickinson). The microorganism was identified by mass spectrometry (MALDI-TOF MS, Bruker Biotyper, Billerica, MA, USA) as E. miricola (Log score 2.3). The MIC of different antibiotics was carried out by the MicroScan device (Beckman Coulter). According to the breakpoints of CLSI for non-fermenting Gram-negative rods, E. miricola was susceptible to ciprofloxacin (MIC $0.38 \mathrm{mg} / \mathrm{L}$ ), levofloxacin (MIC $0.25 \mathrm{mg} / \mathrm{L}$ ), piperacillin-tazobactam (MIC $16 \mathrm{mg} / \mathrm{L}$ ) and resistant to amikacin (MIC>256 mg/L), cefepime (MIC>256 mg/L), ceftazidime (MIC>256 mg/L), colistin (MIC>256 mg/L), fosfomycin (MIC>256 mg/L), gentamicin (MIC $32 \mathrm{mg} / \mathrm{L}$ ), imipenem $(\mathrm{MIC}>32 \mathrm{mg} / \mathrm{L})$, meropenem $(\mathrm{MIC}>32 \mathrm{mg} / \mathrm{L})$ and tetracycline (MIC >24 mg/L). MIC to tigecycline was $0.5 \mathrm{mg} / \mathrm{L}$. At this stage, antimicrobial treatment was started with piperacillin-tazobactam $4 \mathrm{gr} / 8 \mathrm{~h}$ i.v, but after 15 days of treatment the patient had poor renal function without a known cause, and it was decided to change the treatment to oral levofloxacin $500 \mathrm{mg} / 24 \mathrm{~h}$ for three weeks. The patient presented good evolution and after going to successive consultations, 4 months later she was definitively discharged.

At this time, the most common microorganisms causing bone infection are staphylococci or Gram-negative bacilli, including Pseudomonas aeruginosa. From cultures of intraoperative specimens, Staphylococcus aureus is the main causative agent of chronic bacterial osteomyelitis, accounting for about two thirds of isolates, followed by Pseudomonas and Enterobacterales [6]. However, other rare microorganisms could be implicated in the etiology of these infections, as in our case, so physicians and microbiologist should be aware about this possibility. 
The first case of human infection due to E. miricola was reported in 2008 in an adult with mantle cell carcinoma who underwent stem cell transplantation [3]. After this, E. miricola has demonstrated to cause bacteremia, sepsis, and pulmonary and tract urinary infections $[3-5,7]$. From these patients, three of them had underlying comorbidities such as cancer, alcoholic pancreatitis and cystic fibrosis. In all cases, the isolate was identified by MALDI-TOF MS. Thus, the recent introduction of mass spectrometry for routine identification in the clinical laboratories may help to identify some rare pathogens and to know the true incidence of infections due to these microorganisms.

Currently, there are no CLSI/EUCAST guidelines for E. miricola. This bacterium has been found to be multidrug resistant, similar to E. meningoseptica which is known to harbor $\beta$-lactamases showing resistance to $\beta$-lactams and carbapenems. $E$. miricola isolates have been found to be resistant to many antibiotics. A study showed resistance to imipenem, ceftazidime, cotrimoxazole and variable susceptibility to quinolones [8]. In a recent study, all isolates of $E$. miricola were susceptible to tetracyclines and piperacillin-tazobactam. However, 50\% of the isolates were susceptible to levofloxacin and tigecycline [9]. Another study showed that $91 \%$ and $77 \%$ of Elizabethkingia spp isolates were resistant to ciprofloxacin and levofloxacin, respectively [10]. The most prevalent alterations were two single mutations in GyrA, Ser83le and Ser83Arg. In our case, however, the isolate was susceptible to both levofloxacin and ciprofloxacin.

In summary, we here presented the first case of bone infection due to E. miricola. Until now, the presence of this pathogen is rare as cause of human infections, but the recent introduction of MALDI-TOF MS can help to identify some microorganisms, as in our case, which rarely produces bone infections. On the other hand, susceptibility to these isolates should be performed due to the fact that several species often exhibit extensive antimicrobial resistance.

\section{FUNDING}

None to declare.

\section{CONFLICTS OF INTEREST}

The authors declare that there are no conflicts of interest.

\section{REFERENCES}

1. Li Y, Kawamura Y, Fujiwara N, Naka T, Liu H, Huang X et al. Chryseobacterium miricola sp. nov., Novel Species Isolated from Condensation Water of Space Station Mir System. Appl Microbiol 2003; 26: 523-528. DOI: 10.1078/072320203770865828

2. Kim KK, Kim MK, Lim JH, Park HY, Lee ST. Transfer of Chryseobacterium meningosepticum and Chryseobacterium miricola to Elizabethkingia gen. nov. as Elizabethkingia meningoseptica comb. nov. and Elizabethkingia miricola comb. nov. Int J Syst Evol Microbiol 2005; 55: 1287-93. DOI: 10.1099/ijs.0.63541-0
3. Green O, Murray P, Gea-Banacloche JC. Sepsis caused by Elizabethkingia miricola successfully treated with tigecycline and levofloxacin. Diagn Microbiol Infect Dis 2008; 62: 430-432. DOI: 10.1016/j. diagmicrobio.2008.07.015

4. Rossati A, Kroumova V, Bargiacchi O, Brustia D, Luigi Garavelli P. Elizabethkingia miricola bacteremia in a young woman with acute alcoholic pancreatitis. Presse Med 2015; 44: 1071-1072. DOI: 10.1016/j.lpm.2015.08.003

5. Frost F, Dilip N. Case report: First report of Elizabethkingia miricola infection in a patient with cystic fibrosis. Version 2. F1000Res. 2018; 7: 440. DOI: 10.12688/f1000research.14441.2

6. Gross T, Kaim AH, Regazzoni P, Widmer AF. Current concepts in posttraumatic osteomyelitis: a diagnostic challenge with new imaging options. J Trauma 2002; 52: 1220-1219. DOI: 10.1097/00005373200206000-00032

7. Gupta P, Zaman K, Mohan B, Taneja N. Elizabethkingia miricola: A rare non-fermenter causing urinary tract infection. World J Clin Cases 2017; 5: 187-190. DOI: 10.12998/wjcc.v5.i5.187

8. Han MS, Kim H, Lee Y, Kim M, Ku NS, Choi JY, et al. Relative prevalence and antimicrobial susceptibility of clinical isolates of Elizabethkingia species based on 16S rRNA gene sequencing. J Clin Microbiol 2017; 55: 274-280. DOI: 10.1128/JCM.01637-16

9. Chen $Y H$, Perng $C L$, Jian MJ, Cheng YH, Lee SY, Sun JR, et al. Multicentre study evaluating matrix-assisted laser desorption ionization-time of flight mass spectrometry for identification of clinically isolated Elizabethkingia species and analysis of antimicrobial susceptibility. Clin Microbiol Infect 2019; 25: 340-345. DOI: 10.1016/j. cmi.2018.12.005

10. Jian MJ, Cheng YH, Perng CL, Shang HS. Molecular typing and profiling of topoisomerase mutations causing resistance to ciprofloxacin and levofloxacin in Elizabethkingia species. PeerJ 2018; 6: e5608. DOI: 10.7717/peerj.5608. 